\title{
PRIMER REGISTRO DE LEUCISMO PARCIAL EN UNA NIDADA \\ DE TIARIS OLIVACEUS (PASSERIFORMES: THRAUPIDAE) DEL OCCIDENTE DE CUBA
}

\section{First record of partial leucism in a nest of Tiaris olivaceus (Passeriformes: Thraupidae) from Western Cuba}

\begin{abstract}
Jorge Luis Guerra Solana ${ }^{1, *}$, Dayron Breto Benítez ${ }^{2 a}$, Derick Breto Benítez ${ }^{2 b}$
${ }^{1}$ División de Colecciones Zoológicas, Instituto de Ecología y Sistemática, CITMA. Carretera de Varona No. 11835, entre Oriente y Lindero, Reparto Parajón, Municipio Boyeros, La Habana 19, C.P. 11900, Cuba; (1) orcid.org/00000002-1318-4932. ${ }^{2}$ Calle 33 \#3405 e/ 34 y 36, San Diego de los Baños, Los Palacios, Pinar del Río, C.P. 22900, Cuba; 2a (1) orcid.org/0000-0002-0027-6408, ${ }^{2 b}$ (1) orcid.org/0000-0002-0784-3794. "Para correspondencia: guerra@ecologia.cu.
\end{abstract}

\section{RESUMEN}

Se describe un caso de leucismo parcial observado en un individuo de una nidada de tres pichones de Tiaris olivaceus (Linnaeus, 1766). El pichón mostró una coloración blanco-amarillenta en las plumas de la parte superior de la cabeza, alas y cola, conservando el patrón típico de coloración en el resto del cuerpo. En la literatura científica, y las principales colecciones de aves del país, no existen registros previos de leucismo para la región. Los avicultores y pobladores locales de la zona del hallazgo, no refieren ningún otro caso de aves con coloración atípica, por lo que concluimos que el leucismo sigue siendo un evento muy raro dentro de las poblaciones de T. olivaceus del área.

Palabras clave: Tomeguín de la tierra, leucismo parcial, nidificación, Cuba.

\section{ABSTRACT}

Partial leucism in one of three nestling of a brood of Yellow-faced Grassquit, Tiaris olivaceus (Linnaeus, 1766), is described. Young bird had a white-yellowish coloration in its primary wings feather, rectrix and crown. Rest of the body remained with its natural coloration. In the scientific literature, and major bird collections of Cuba, there are no previous records of leucism for the region. Also, there are no references from local residents and aviculturist to this untypical pattern before. We conclude that leucism is a very rare event inside T. olivaceus' populations of the area.

Keywords: Yellow-faced Grassquit, partial leucism, nidification, Cuba.

El color del plumaje en las aves, es primariamente el resultado de la deposición de una combinación de pigmentos (melaninas, carotenoides y porfirinas) en las plumas. La manera en la que estos pigmentos se distribuyen, así como la interacción de la luz con la microestructura de las plumas, da como resultado la diversidad en patrones de coloración (Auber, 1957; Clark, 2004).

Si algún pigmento no se incorpora ordinariamente durante el desarrollo de la pluma, se obtienen como consecuencia aberraciones en la coloración del plumaje. Estas aberraciones pueden tener un origen genético o ser resultado de presiones ambientales como una dieta deficiente o enfermedades. Las más frecuentes en aves de vida libre son: albinismo, leucismo, esquizocroísmo y dilución (van Grouw, 2013). 
En las aves albinas, la ausencia de pigmentos se da tanto en las plumas como en los tejidos del cuerpo; mientras que en las aves con leucismo, solo ocurre una reducción significativa de los pigmentos en las plumas y algunas partes de la piel. En consecuencia, los ojos de un ejemplar leucístico siempre conservarán su color natural (a diferencia del ojo rojo de uno albino). Las patas y el pico, en cambio, pueden presentarse de color rosado. El leucismo parcial, es el más frecuente en aves de vida libre (McCormac, 2001; van Grouw, 2006; Guay et al., 2012).

El Tomeguín de la tierra (Tiaris olivaceus) es un paserino de pequeño tamaño cuyas poblaciones se distribuyen desde el sureste de México hasta el norte de Suramérica, las Antillas Mayores e Islas Caimán. Prefiere hábitats abiertos, y es común observarlo en matorrales, ecosistemas agrícolas y el borde de caminos y bosques (Torok y Burns, 2011).

Es una especie con dicromatismo sexual, siendo los machos de colores más brillantes y llamativos que las hembras. En ambos sexos el cuerpo es mayormente de color olivo por encima y grisáceo por debajo. Los machos muestran, en las cejas y el parche de la garganta, una coloración amarillo-naranja, además de una mancha negra en el pecho que aumenta de tamaño con la edad. Las hembras, en cambio, presentan la coloración de las cejas y la garganta amarillo claro, y carecen del parche negro en el pecho (Garrido y Kirkconnell, 2011).

Las aberraciones en la coloración del plumaje de $T$. olivaceus son poco comunes en el ambiente natural, donde se han encontrado aves completamente blancas, con coloración amarillenta extendida o con partes del plumaje manchado de blanco. Las primeras observaciones de estos patrones de coloración inusuales en Cuba datan del siglo XIX, y no precisan las localidades de los hallazgos (Gundlach, 1876). Se tiene referencias, además, de un caso concreto de xantismo para la especie (Moreno, 1946).

Posterior a estas observaciones, se han documentado en el país cuatro registros de leucismo parcial para la especie. Estos reportes se circunscriben a la región centro-oriental; el primer avistamiento fue realizado en la cayería norte de Ciego de Ávila (Kirwan, 2001) y los restantes en Camagüey (Acosta 2005, 2007). Cabe destacar, además, que no existen especímenes leucísticos de T. olivaceus en las principales colecciones científicas cubanas: Instituto de Ecología y Sistemática, Museo Felipe Poey y Museo Nacional de Historia Natural.

En este trabajo, documentamos la presencia de un pichón leucístico en una nidada de Tomeguín de la tierra localizada en San Diego de los Baños (Pinar del Río, $22^{\circ} 39^{\prime} \mathrm{N}$ y $83^{\circ}$ 22' O, Municipio Los Palacios) el 4 de junio de 2015. El pichón, con leucismo parcial, presentaba toda la cabeza blanco-amarillenta, así como las primarias de ambas alas y las plumas rectrices. La parte final del vientre tendía a un gris más claro de lo habitual, y de manera general, el ave sólo conservaba el color natural en el pecho, dorso, ojos, patas, parte de la cara y el pico (Fig. 1). La nidada era de tres pichones, y los otros dos hermanos presentaban coloración normal. El pichón leucístico era el de mayor tamaño y lamentablemente su coloración llamativa hizo que toda la nidada fuese capturada por un lugareño para su cría en cautiverio.

El presente registro constituye el primer caso de leucismo parcial en T. olivaceus para el occidente de Cuba. Hasta el momento, las causas que producen este tipo de aberración cromática en la especie, y su efecto en la supervivencia de los individuos afectados continúan sujetas al estudio. Cabe destacar que los avicultores y pobladores locales de la zona del hallazgo, no refieren ningún otro caso de tomeguines con coloración atípica, por lo que concluimos que el leucismo sigue siendo un evento muy raro dentro de las poblaciones de $T$. olivaceus del área. 


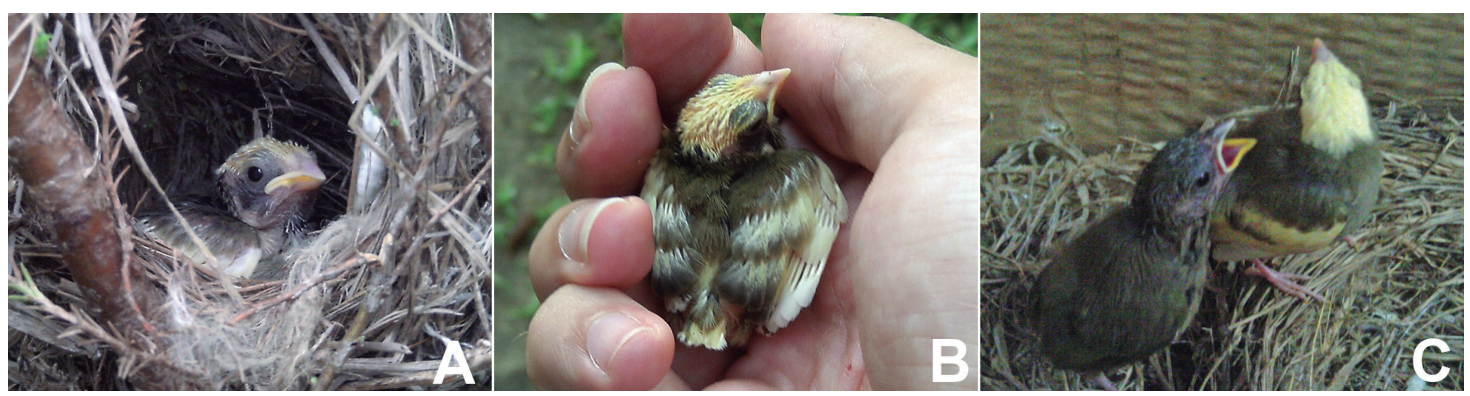

Figura 1. Pichón de Tiaris olivaceus con leucismo parcial, encontrado en una nidada de tres pichones en San Diego de los Baños, Cuba. A, en el nido; B, vista dorsal; C, pichón leucístico junto a uno de sus hermanos de coloración normal. Fotografías de Dayron Breto.

\section{AGRADECIMIENTOS}

Agradecemos a Ianela García (Museo Felipe Poey) y Xochitl Ayón (Museo de Historia Natural) por facilitar la información de las colecciones de aves. A Ormaily Madruga y dos revisores anónimos por sus valiosos comentarios al manuscrito inicial.

\section{LITERATURA CITADA}

Acosta, L. 2005. Primer caso conocido de leucismo parcial en Tiaris olivacea en Cuba. Revista Mexicana de Ornitología, 6 (2): 14-15.

Acosta, L. 2007. Tres casos de leucismo en Tiaris olivaceus: Una rara coincidencia en la ornitofauna de Camagüey, Cuba. Ornitología Colombiana, 5: 81-82.

Auber, L. 1957. The distribution of structural colours and unusual pigments in the Class Aves. Ibis, 99: 463-476.

Clark Jr., G. A. 2004. Form and function: The external bird. Capítulo 3. En: Podulka, S., R. W. Rohrbaugh Jr. y R. Bonney, (Eds.), Handbook of Bird Biology. The Cornell Lab of Ornithology, Ithaka, New York.

Garrido, O. H. y A. Kirkconnell. 2011. Aves de Cuba. Cornell University Press. Ithaka, New York., 287 pp.

Guay, P. J., D. A. Potvin y R. W. Robinson. 2012. Aberrations in plumage coloration in birds. Australian Field Ornithology, 29: 23-30.

Gundlach, J. 1876. Contribución a la ornitología cubana. Academia de Ciencias de Cuba, La Habana, 369 pp.

Kirwan, G. M. 2001. A leucistic Yellow-faced Grasquit (Tiaris olivacea) in Cuba. The Journal of Caribbean Ornithology, 14 (1): 10.

McCormac, J. 2001. Albinism in birds. The Ohio Cardinal, 25 (1): 36-39.

Moreno, A. 1946. Notas ornitológicas. Memorias de la Sociedad Cubana de Historia Natural, 18: $186-187$. 
Torok, M. y K. J. Burns. 2011. Yellow-faced Grassquit (Tiaris olivaceus), version 1.0. En: T. S. Schulenberg, editor. Neotropical Birds Online. Cornell Lab of Ornithology, Ithaca, New York. https://doi.org/10.2173/nb.yefgra1.01.

Van Grouw, H. 2006. Not every white bird is an albino: sense and nonsense about colour aberrations in birds. Dutch Birding, 28: 79-89.

Van Grouw, H. 2013. What colour is that bird? The causes and recognition of common colour aberrations in birds. British Birds, 106: 17-29.

[Recibido: 6 de noviembre, 2019. Aceptado para publicación: 26 de marzo, 2020] 